\title{
La seguridad de medidores de agua potable contra robo, vandalización y manipulación - problemática,
} avances y propuesta

\section{Security of potable water meters against theft, vandalism and tampering - problems, advances and proposal}

\author{
Stefan Ziemendorff ${ }^{1 *}$, Wagner Vásquez Velásquez y Hermes Ruesta Córdova ${ }^{2}$
}

\section{RESUMEN}

Uno de los principales problemas de las entidades prestadoras de servicios de agua potable y saneamiento consiste en la manipulación, la vandalización y el robo de los medidores de consumo de agua, resultando en pérdidas económicas importantes, tanto por la subfacturación del consumo como por el costo del reemplazo de los medidores. Por ello, en primer lugar, el presente trabajo analiza la casuística de las diferentes formas de manipulación y destrucción de los medidores de agua. Luego, sobre la base de los resultados de este análisis, se definen los puntos vulnerables del medidor que deben ser protegidos. Asimismo, se realiza una evaluación de los dispositivos de seguridad existentes, la cual demuestra que ninguno de ellos protege de forma eficaz todos los puntos débiles del medidor. Finalmente, se proponen dos nuevos dispositivos de seguridad para los medidores y se detalla su modo de instalación, así como los resultados de una instalación piloto de una muestra de estos dispositivos en la ciudad de Talara (Perú), concluyendo que brindan una protección mucho mayor contra el robo y la destrucción que todos los dispositivos usados hasta ahora.

Palabras clave: Dispositivos de seguridad de medidores de agua, manipulación de medidores de agua.

\begin{abstract}
One of the main problems of water and sanitation service providers is the tampering, vandalism and theft of water consumption meters, resulting in significant economic losses, both due to the under-billing for consumption and the cost of meter replacement. First, the present work analyzes the variety of different forms of manipulation and destruction of water meters. Then, based on the results of this analysis, the vulnerabilities of the meters that need to be protected are defined. An evaluation of the existing safety devices is performed, which shows that none of them effectively protect all the vulnerabilities of the meters. Finally, two new meter safety devices are proposed and their installation proceduresare detailed, as well as the results of a pilot installation of a representative example of these devices in the city of Talara (Peru). The paper concludes that these meter safety devices provide a much greater protection against theft and destruction than all the previous devices in current use.
\end{abstract}

Keywords: Water meter security devices, tampering of water meters

\footnotetext{
'AKUT PERÚ S.A.C. Programa de Modernización y Fortalecimiento del Sector Agua y Saneamiento - PROAGUA 11, Deutsche Gesellschaft für Internationale Zusammenarbeit (GIZ) GmbH, Av. Los Incas 172, Piso 5 - El Olivar, San Isidro - Lima 27, Perú.

${ }^{2}$ Banco de medidores EPS GRAU S.A. Esquina Jr. La Arena y Jr. Zelaya S/N - Urb. Santa Ana, Jr. La Arena s/n, Piura, Perú

"Autor de correspondencia. E-mail: sziemendorff@gmail.com
} 


\section{INTRODUCCIÓN}

Desde la invención del primer medidor de agua apto para uso masivo por Carl Wilhelm Siemens en 1851, que luego fue usado de forma masiva, las entidades prestadoras del servicio de agua potable han lidiado con dos problemas: el correcto funcionamiento de los medidores para evitar sub-y sobre-registros, y la manipulación de los equipos por parte de usuarios que tratan de aminorar la facturación de agua consumida. En varios países se ha tratado de dar soluciones diferentes a este último problema; por ejemplo, es por este motivo que en varios países de Europa el medidor se encuentra en el interior de la vivienda del consumidor, de modo que cualquier manipulación del dispositivo es automáticamente atribuida a él y, por ende, judicialmente perseguida.

Sin embargo, en la mayoría de los países del mundo, entre ellos los de América Latina, los medidores suelen encontrarse en la vía pública, lo que facilita la lectura mensual, pero al mismo tiempo causa problemas en el momento de encontrar un medidor manipulado, robado o vandalizado, ya que es prácticamente imposible comprobar la autoría de la manipulación. En el caso del Perú, se ha tratado de encontrar un punto intermedio entre la imposibilidad de atribuir el acto de manipulación y la necesidad de tomar medidas que desincentiven la manipulación. Así, se le cobra el costo del medidor y su instalación al consumidor en el caso de encontrar un medidor alterado por segunda vez en un periodo de cinco años (SUNASS, 2007).

En el marco del proyecto "Agua para la vida", financiado por la Cooperación Suiza y administrado por la EPS Grau S.A. en la costa norte del Perú, se intervino en las provincias de Paita (130000 habitantes y 20000 conexiones de agua potable, aproximadamente) y Talara (135000 habitantes y 30000 conexiones de agua potable, aproximadamente), entre otras con la finalidad de disminuir las pérdidas comerciales.

Entre los problemas mayores detectados durante la intervención, se identificó la gran cantidad de medidores manipulados: los datos del banco de medidores de la EPS Grau S.A. mostraron que entre el 2008 y media- dos del 2016, 929 medidores en Talara, y 604 en Paita, fueron manipulados. Por estas razones, el objetivo de nuestro proyecto consiste en hallar una solución a este problema.

\section{MATERIALY MÉTODOS}

Para poder proponer una solución viable al tema de la manipulación de medidores, se implementaron los siguientes pasos:

1) Se analizó la casuística de las manipulaciones de medidores, usando la información del banco de medidores de la EPS Grau.

2) Se realizó la clasificación de los casos analizados por tipo de manipulación.

3) Se identificaron los puntos vulnerables del medidor.

4) Se analizó la frecuencia de cada tipo de manipulación en Paita y Talara.

5) Se analizaron los dispositivos de seguridad disponibles en cuanto a la protección que brindan a los diferentes puntos vulnerables identificados.

6) Se identificó la necesidad de desarrollar dispositivos de seguridad más integrales.

7) Se desarrollaron dos prototipos de seguridad del medidor.

\section{RESULTADOS Y DISCUSIÓN}

\section{Tipos de manipulación}

El hurto del agua potable tiene varias causas y muchísimas formas de manifestarse, lo que requiere una diversificación de los métodos para prevenir y combatirlo. Entre los motivos para hurtar agua figuran la dificultad de conseguirla de forma legal, ya sea por los costos o trámites que la vía legal implica (Alva Vives et al., 2006a; Cubas Rojas et al., 2006), o por el hecho de no poder o no querer pagar el agua (Alva Vives et al., 2006b). La manipulación de la lectura del medidor con la finalidad de disminuir la facturación figura entre los escenarios encontrados con mayor frecuencia y es particularmente difícil de combatir. La casuística correspondiente detectada en Paita y Talara evidencia 
un gran espectro de tipos de manipulación. El análisis exhaustivo de tipos de hurto de agua en general se hizo en otro área del país (Ziemendorff, 2015), así que en este caso solamente se describirán aquellas formas de hurto que tienen como finalidad alterar el consumo registrado. Existen tres maneras de hacerlo, cada una con varias modalidades; resaltaremos las que son de relevancia para el tema presentado:

1) Dejar el medidor en condiciones perfectas sin tocarlo, pero evitar el pase del agua mediante la instalación de una conexión clandestina adicional, un bypass o una conexión vía un vecino sin medidor. Sobre la detección de este tipo de instalaciones clandestinas véase Ziemendorff (2015).

2) Dejar el medidor en pleno funcionamiento, de tal forma que, al inspeccionarlo, no es posible detectar la manipulación. Se realiza de las siguientes maneras:

a) Inversión del medidor: Consiste en voltear el medidor para que en vez de aumentar, la lectura disminuya conforme sigue el consumo. Posteriormente se coloca en su posición normal para que el lecturista no se dé cuenta de la manipulación. En el caso de que el usuario se olvide de colocar el medidor a tiempo a su posición normal, a veces se detecta la manipulación si el consumo del mes resulta ser negativo, siendo la lectura del mes actual más baja que la del mes anterior.

b) Extracción temporal del medidor: En periodos de muy alto consumo, se extrae el medidor; pasado este periodo, se vuelve a instalar.

c) Retroceder la lectura del medidor: Es un tipo de manipulación parecida a la inversión del medidor, pero en este caso se usa un flujo de aire a presión, como el que sirve para inflar las llantas de autos. Justamente por ello, este tipo de manipulación se encuentra con cierta frecuencia en lavaderos de vehículos. Después de la manipulación se vuelve a instalar el medidor. Esta forma es muy difícil de detectar debido al corto tiempo por el que se extrae el medidor.

3) Alterar el medidor para que deje de funcionar parcial o totalmente:

a) Manipulación no visible: El consumidor retira o desgasta algunos dientes del engranaje del registro del medidor, desgasta las hélices de la turbina del medidor u obstaculiza la transmisión magnética de su movimiento a la relojería con un chip de plástico, de modo que el medidor mida un consumo mucho menor que el real. Para esto es necesario retirar o bien el medidor completo o solo el registro. Aunque este es protegido por un anillo que a la vez cuenta con un precinto de seguridad numerado, se ha constatado que es posible retirar y volver a instalarlo sin vulnerar el precinto.

b) Vandalización total (destrucción): Consiste en el uso de fuerza bruta para conseguir que el medidor deje de funcionar o ya no pueda ser leído, golpeándolo desde arriba con una barreta hasta destruir la relojería.

c) Perforación de la luneta del medidor: Consiste en destruir el reloj del medidor perforándolo en la parte del engranaje con una aguja caliente. En ocasiones los huecos ocasionados son tan diminutos que no saltan a la vista del lecturista.

d) Rayar la luneta del medidor: Consiste en dejar el medidor en función, pero hacer imposible la lectura al rayar la luneta plástica.

e) Introducción de objetos paralizadores por el tubo: Consiste en introducir un alambre $u$ otro objeto largo y rígido por el tubo de agua hasta la turbina del medidor. Normalmente se requiere temporalmente retirar el medidor para ello.

f) Auto-robo del medidor: El cliente extrae su 
propio medidor. A pesar de la facilidad de detectar el caso, mayormente no se puede evidenciar que el robo fue cometido por el mismo cliente.

g) Robo del registro (relojería): El cliente roba solamente el registro del medidor.

Algunas de estas formas de manipulación se muestran en la figura 1.
Frecuencia de los tipos de manipulación encontrados en Paita y Talara

Entre 2008 y 2016 se registraron en el banco de medidores de EPS Grau 1563 casos de manipulación de medidores provenientes de Paita y Talara. No se cuenta ni con una estadística de medidores robados, extraídos temporalmente o retrocedidos para el periodo

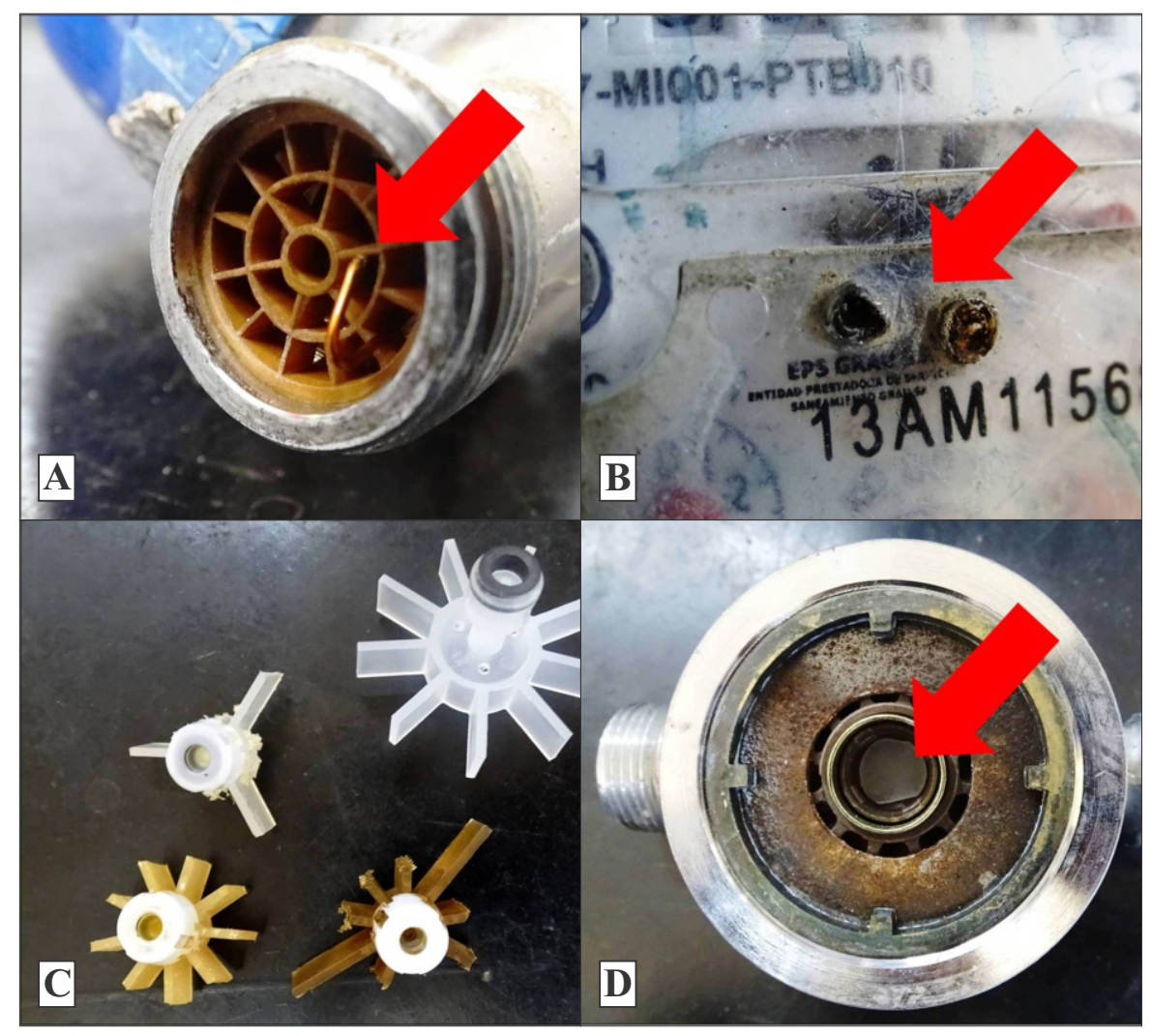

Figura 1. A. Un alambre de cobre introducido por el filtro para paralizar la hélice; B. Dos perforaciones en la luneta de la relojería para destruir el engranaje; C. Varias hélices recortadas para frenar la rotación. En la parte superior derecha se muestra una hélice normal; D. Un chip de plástico introducido en la carcasa para frenar la transmisión magnética de la hélice a la relojería.

2008-2016, ni con datos para el mismo periodo referente al tercer tipo de manipulaciones (conexiones clandestinas adicionales y bypass), debido a que no son casos registrados por el banco de medidores. Las 1563 incidencias de manipulaciones registradas se distribuyeron de la forma mostrada en la figura 2.

\section{Puntos vulnerables del medidor}

Partiendo de la casuística se ha encontrado que el medidor de agua tiene seis puntos propensos a la manipulación, la vandalización o el retiro, puntos en los cuales debe ser protegido para asegurar su correcto funcionamiento (ver figura 3).

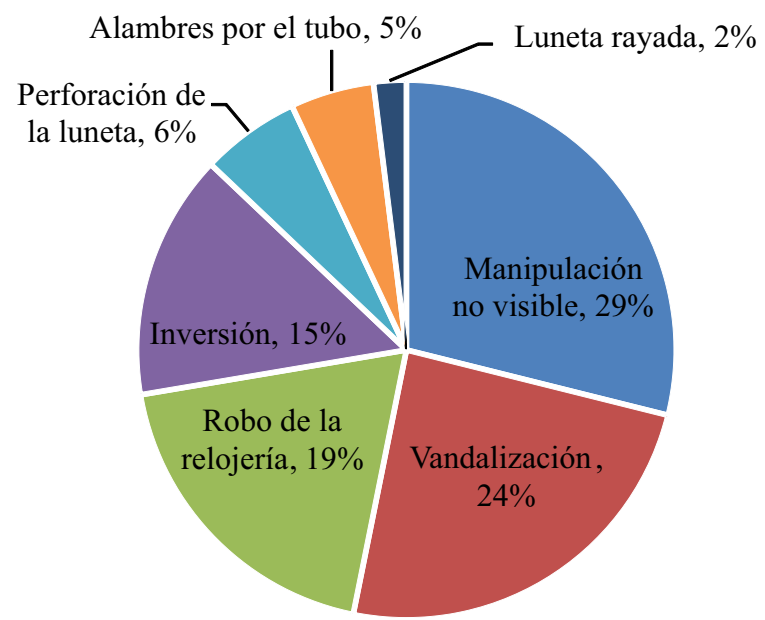

Figura 2. Distribución de las incidencias en los medidores. 


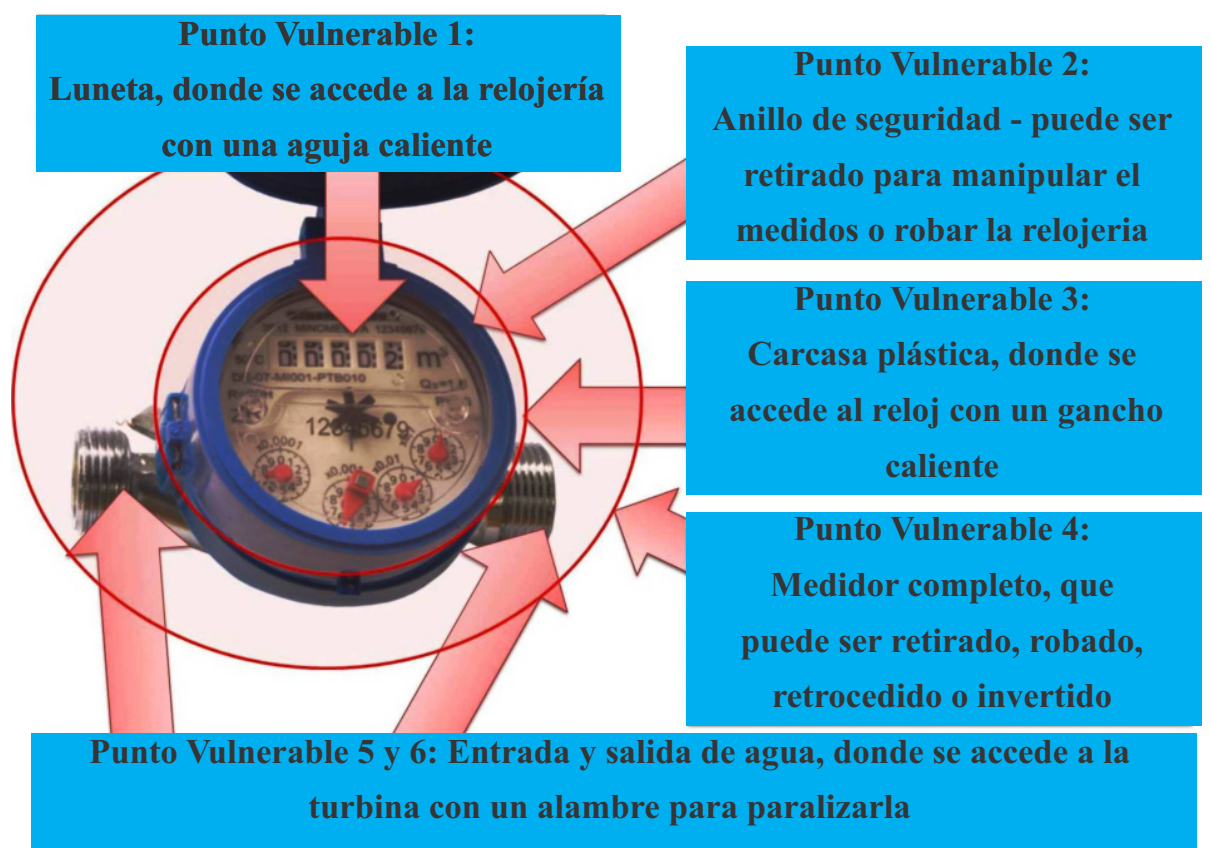

Figura 3. Los seis puntos vulnerables de un medidor de agua potable y las variedades correspondientes de alteración del equipo.

Análisis de dispositivos de seguridad actualmente

\section{disponibles para medidores}

Un primer análisis de dispositivos de seguridad para medidores, efectuado hace algunos años (Alva Vives,
2009) resulta incompleto hoy en día, debido a las numerosas invenciones hechas en el campo entretanto. Aquí presentaremos una selección representativa de estas (ver figura 4).

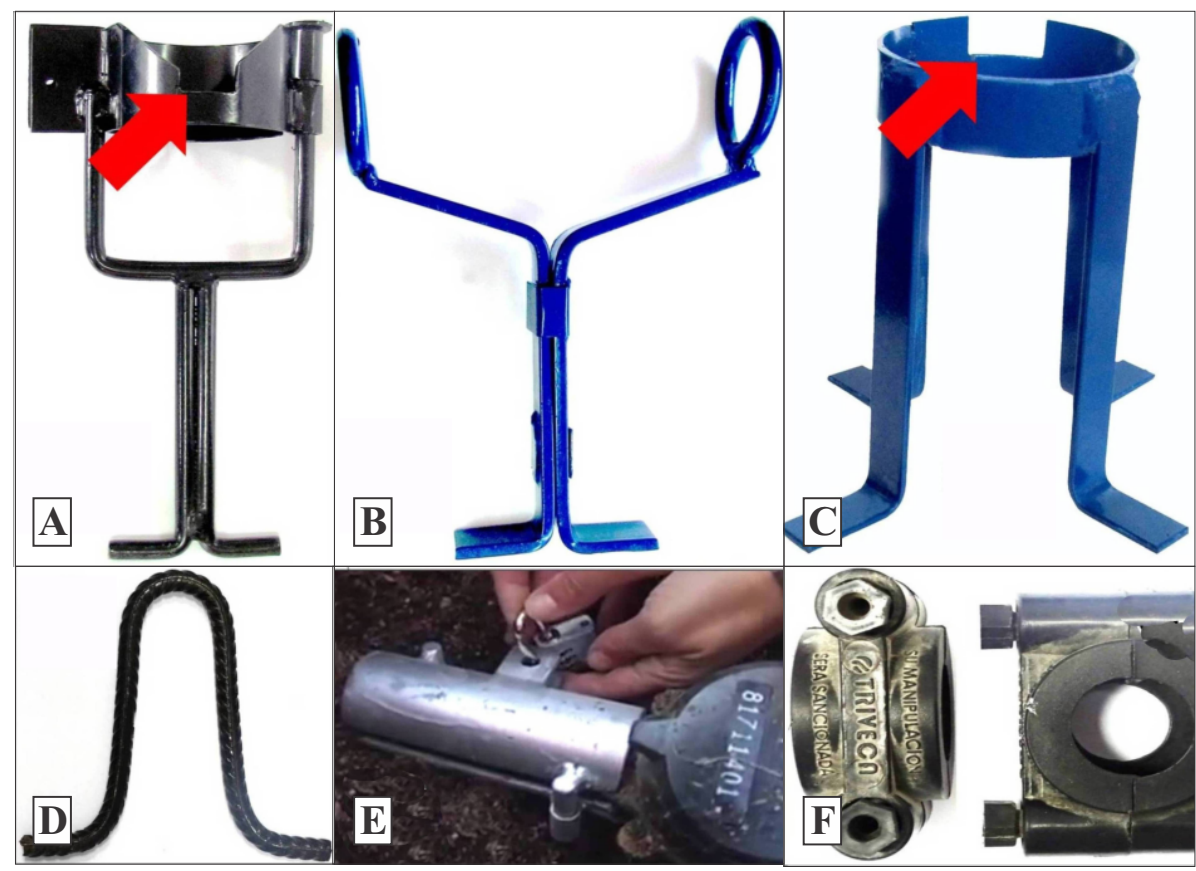

Figura 4. A. Anclaje Tipo Copa (Medileser, 2011a), la flecha roja indica la parte de la cinta de protección prevista para las bisagras de la tapa del medidor; B. Anclaje Tipo Argolla (Medileser, 2011b); C. Anclaje Tipo Pulpo (Medileser, 2011c), la flecha roja indica la parte de la cinta de protección prevista para las bisagras de la tapa del medidor; D. Anclaje Tipo Gauss, se instalan uno por cada lado del medidor; E. Water Meter Lock (Danger, 2012); F. Precinto de seguridad de plástico; también existen productos de metal. 
Respecto a la instalación de los dispositivos de la figura 4, los tipos A-D son empotrados en el fondo de la caja del medidor, mientras los demás (E y F) fijan el medidor en el tubo. En cuanto a su utilidad y nivel de seguridad presentan grandes diferencias (Tabla 1).

Tabla 1. Cuadro comparativo de los dispositivos de seguridad según la protección que brindan al medidor de agua en los seis puntos vulnerables previamente identificados

\begin{tabular}{|c|c|c|c|c|c|}
\hline \multirow{2}{*}{ NOMBRE DEL DISPOSITIVO } & \multicolumn{5}{|c|}{ PROTECCIÓN DEL PUNTO VULNERABLE } \\
\hline & 1 & 2 & 3 & 4 y 5 & 6 \\
\hline A - Anclaje Tipo Copa & No & Sí & $\begin{array}{c}\text { No, pero se } \\
\text { podría adecuar }\end{array}$ & Sí & Sí \\
\hline B - Anclaje Tipo Argolla & No & No & No & Sí & Sí \\
\hline C - Anclaje Tipo Pulpo & No & Sí & $\begin{array}{c}\text { No, pero se } \\
\text { podría adecuar }\end{array}$ & Sí & Sí \\
\hline D - Anclaje Tipo Gauss & No & No & No & Sí & Sí \\
\hline E - Water Meter Lock & No & No & No & Sí & Sí \\
\hline F - Precintos & No & No & No & Sí & Sí \\
\hline
\end{tabular}

La tabla muestra que todos los dispositivos protegen el medidor contra el retiro completo y la introducción de objetos paralizadores. Pero solo los dispositivos de seguridad más completos - el Anclaje Tipo Copa (A) y el Anclaje Tipo Pulpo (C) - protegen el medidor adicionalmente contra el retiro de la relojería. Además, ambos dispositivos cuentan con una cinta metálica prevista para proteger la carcasa plástica de la relojería. Sin embargo, como se nota en la figura 4, esta cinta presenta una abertura para las bisagras de la tapa del medidor (indicada por las flechas rojas). Esta abertura permite el acceso al medidor con una aguja o gancho caliente con la finalidad de destruir los engranajes de la relojería en una parte difícilmente visible al momento de la lectura. Una opción de superar esta problemática consiste en cerrar la cinta completamente dejando el medidor sin tapa ni bisagras. Pero aun así, quedaría expuesto el punto vulnerable 1 - la luneta plástica del medidor, la cual no está protegida por ninguno de los dispositivos de seguridad disponibles. De todas maneras, cabe resaltar que las diferentes formas de manipulación que se dan por esta vía siempre dejan una evidencia claramente visible: una luneta rayada, perforada o destruida.
Los demás dispositivos (B, D-E) solamente evitan (o dificultan) que el medidor pueda ser retirado, previniendo así el robo, la inversión, la extracción temporal, y la retrocesión del medidor, así como tipos de manipulación laboriosos que requieren sacarlo fuera de la caja. Cabe indicar que, al momento de realizar el presente estudio en Paita y Talara, el dispositivo en uso regular era el Anclaje Tipo Gauss, que se caracteriza por su bajo costo, pero a la vez por su bajo nivel de seguridad. Con su instalación masiva a partir del 2012, aunque sin alcanzar una cobertura total, disminuyeron las incidencias de inversión y de introducción de objetos paralizadores por el tubo, mientras que los demás tipos de manipulación -que no son dificultadas por el dispositivo instalado- aumentaron proporcionalmente (Figura 5).

Esto nos permite llegar a una conclusión altamente relevante: la instalación de dispositivos de seguridad que no protegen todos los puntos vulnerables del medidor a la vez, lleva automáticamente al incremento de las otras modalidades de manipulación, sin efecto sobre el total de manipulaciones. Por ello, se requiere un dispositivo de seguridad que proteja los seis puntos vulnerables del medidor a la vez. 


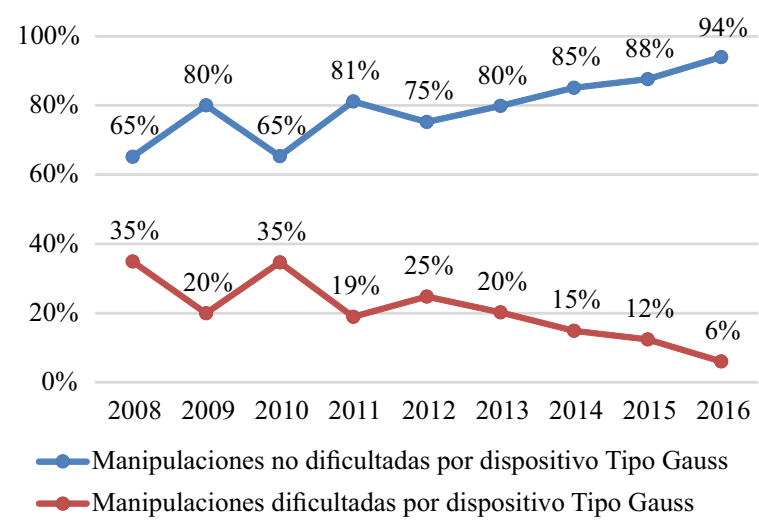

Figura 5. Efecto de la instalación del Dispositivo D (Anclaje Tipo Gauss) en los tipos de manipulación.

\section{Desarrollo de dos nuevos dispositivos}

Sobre la base del análisis de la casuística documentada en Paita y Talara y la identificación de los puntos vulnerables de los medidores, en el marco del mencionado proyecto nos propusimos desarrollar un nuevo tipo de dispositivo de seguridad para medidores. Este nuevo dispositivo deberá cumplir la finalidad de proteger el medidor mejor que todos los dispositivos existentes.

Sin embargo, precisamente esto significa que el nuevo dispositivo tendrá que proteger, además de los puntos asegurados por los dispositivos existentes, los dos puntos restantes expuestos a acciones de manipulación, no protegidos por ninguno de los dispositivos analizados:

1) El costado de la carcasa de la relojería.

2) La luneta.

La solución del primer problema resultó ser bastante sencilla, ya que se presentaron dos opciones simples de proteger el costado de la carcasa: la primera consiste en fabricar un dispositivo que reemplaza la carcasa plástica de la relojería por una cinta metálica no penetrable, quitando la tapa y la bisagra del medidor; de esta manera se obtuvo un dispositivo en forma redonda, igual que el medidor. La otra posibilidad prevé fabricar un dispositivo metálico en el cual entra el medidor sin necesidad de quitar la carcasa; en este caso se obtuvo un dispositivo cuadrado, principalmente debido al hecho de que es más fácil conseguir un tubo metálico cuadrado (Figura 6).
En cambio, la protección de la luneta presentó más obstáculos, principalmente debido a la dificultad de conseguir materiales adecuados en cantidades muy pequeñas para la producción de prototipos. En estos se pensaba colocar un vidrio de seguridad inamovible y resistente a golpes fuertes encima del medidor. El único vidrio disponible a un costo aceptable para la producción del prototipo era el vidrio laminado de 2 vidrios de $5 \mathrm{~mm}$ de grosor, con una lámina de seguridad llamada impenetra de 12 micrones, debajo de la cual se añadió debajo otra capa, hecha de material acrílico transparente.

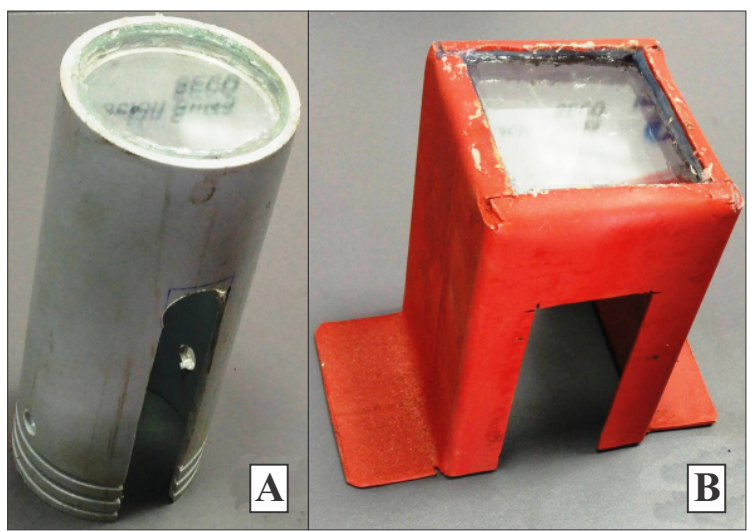

Figura 6. Prototipos de nuevos dispositivos de seguridad para medidores: A. El dispositivo cilíndrico; B. El dispositivo cuadrado.

\section{Descripción de los dos nuevos dispositivos}

El dispositivo cilíndrico (Figura 7) tiene las siguientes características:

- Material: PVC de un diámetro externo de 0,090 m, el cual puede variar en función de la longitud del medidor.

- Espesor del tubo: 0,008 m.

- Altura del dispositivo: 0,210 m; puede variar en función de la profundidad de la caja del medidor.

- Vidrio laminado: consistiendo en dos vidrios pegados de 0,005 $\mathrm{m}$ de grosor cada uno separado por una lámina de seguridad impenetra de 12 micrones. Por debajo del vidrio laminado está pegado un vidrio acrílico adicional de $0,01 \mathrm{~m}$.

- 3 ranuras laterales inferiores de $0,003 \mathrm{~m} \mathrm{c} / \mathrm{u}$ por ambos lados. 


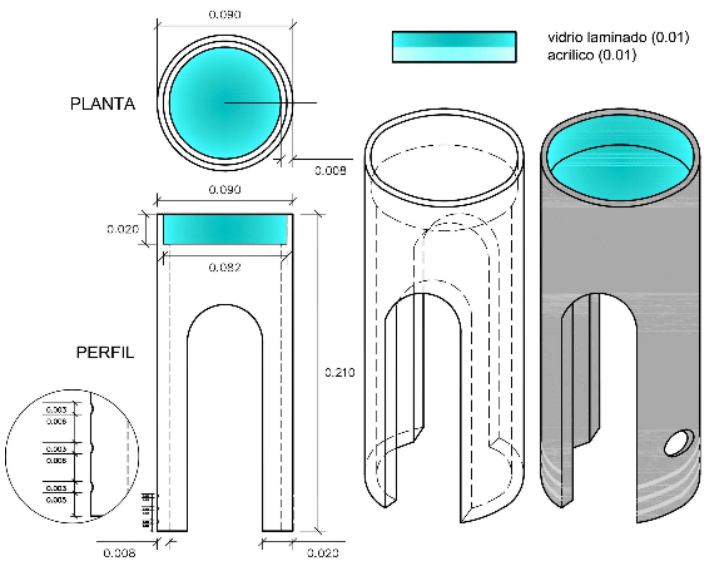

Figura 7. Dibujo isométrico del dispositivo cilíndrico, todas unidades en metros.

El dispositivo cuadrado (Figura 8) tiene las siguientes características:

Material: hierro modelo cuadrado de 0,100 m x 0,100 $\mathrm{m}$; las medidas pueden variar en función de la longitud del medidor.

Espesor del tubo: 0,02 m.

Altura del dispositivo: 0,155 m; puede variar en función de la profundidad de la caja del medidor.

Vidrio laminado: consiste en dos vidrios pegados de $0,005 \mathrm{~m}$ de grosor cada uno separado por una lámina de seguridad impenetra de 12 micrones. Por debajo del vidrio laminado está pegado un vidrio acrílico adicional de $0,01 \mathrm{~m}$.

2 ranuras laterales $(\mathrm{a}=0,050 \mathrm{~m}$ y $\mathrm{h}=0,105 \mathrm{~m})$.

2 pestañas laterales de $0,045 \mathrm{~m}$ para el anclaje.

\section{Instalación experimental de una serie de dispositivos}

En diciembre del 2016, se instalaron 20 ejemplares de los nuevos dispositivos (10 dispositivos cilíndricos y
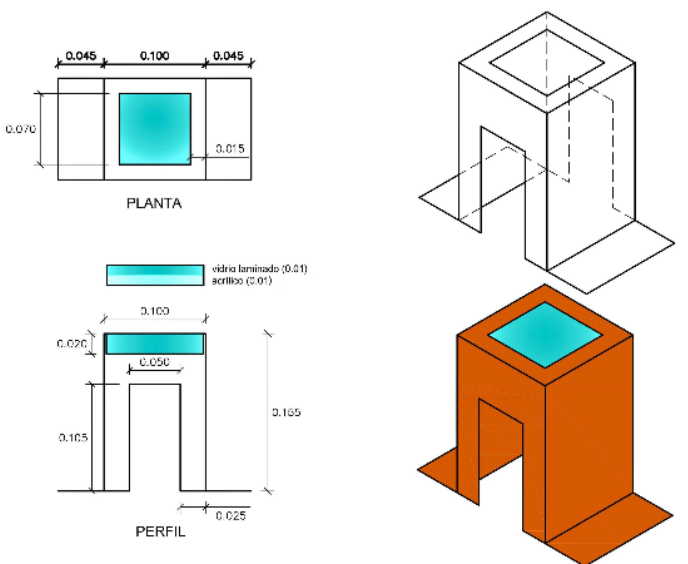

Figura 8. Dibujo isométrico del dispositivo cuadrado, todas las unidades en metros.

10 dispositivos cuadrados) en la ciudad de Talara. Para la instalación se enfocó a clientes que, según la base de datos de la EPS, habían manipulado su medidor de agua entre dos y cuatro veces en los últimos cinco años. En los primeros días del mes de febrero del 2017, se realizó una revisión de los dispositivos instalados que permitió averiguar los hallazgos siguientes (Figura 9):

- Debido a las inusuales lluvias estivales, por un lado, y a la omisión de espuma expansiva en la instalación de los dispositivos, por el otro lado, algunas lunetas se habían empañado, dificultando pero no imposibilitando la lectura del medidor.

- En el caso de un dispositivo cilíndrico, constatamos que la luneta había sido retirada, así como la relojería del medidor. Esta manipulación fue posible gracias al pegamento utilizado (silicona) y la introducción de la luneta desde arriba durante el proceso de elaboración del dispositivo.

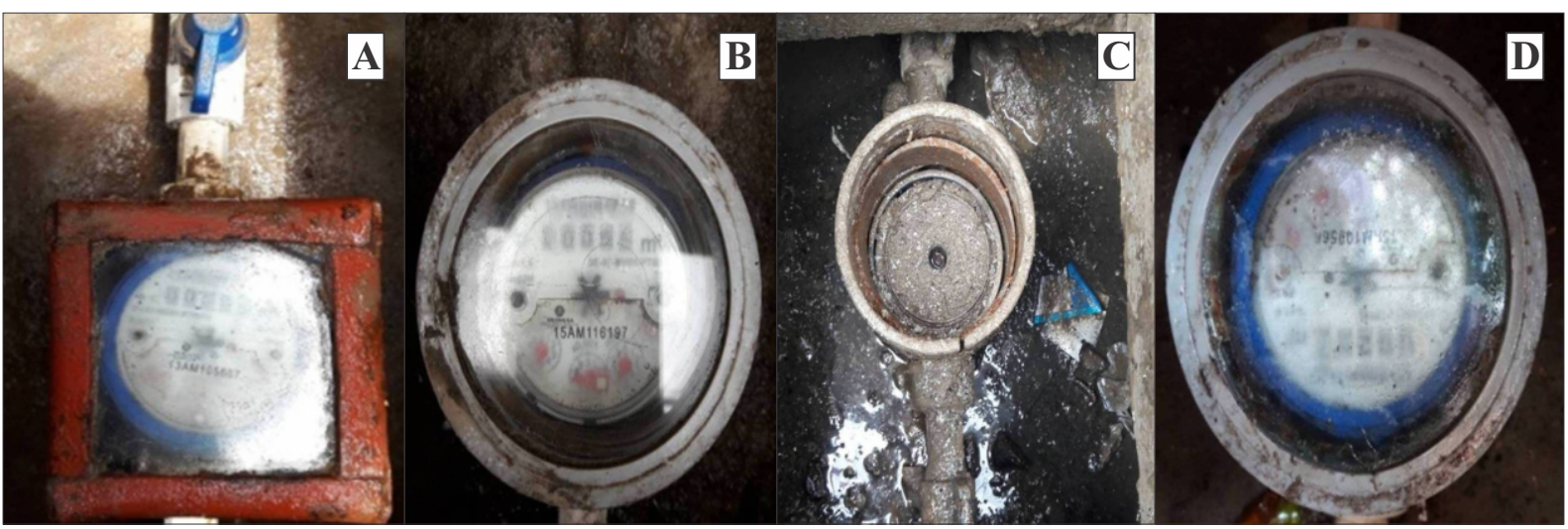

Figura 9. A. Dispositivo cuadrado en campo en estado normal; B. Dispositivo cilíndrico en campo en estado normal; C. Dispositivo cilíndrico y medidor vandalizados; D. Dispositivo cilíndrico con luneta parcialmente empañada. (Fotos: Tania Vega Valle). 
Propuestas de mejora y adecuación de los dispositivos para la producción en serie

Ambos dispositivos:

1) La principal mejora que se propone para ambos dispositivos concierne la luneta de seguridad. Se sugiere usar una luneta compuesta por tres láminas, cada una de un grosor mayor $(8 \mathrm{~mm})$, de manera que se puede prescindir del vidrio acrílico. En el futuro, el uso del vidrio metálico podría constituir otra opción, ya que tendría la ventaja de no poder ser rayado por golpes, de modo que, en el caso de tentativas de manipulación, la luneta quedaría intacta y, por ende, no tendría que ser reemplazada. Sin embargo, este material recientemente ha sido perfeccionado para su uso en celulares y sigue siendo difícil y costoso de conseguir.

2) Asimismo, es recomendable usar otro tipo de pegamento más adecuado con mejores características, con el objetivo de imposibilitar que las lunetas sean removidas del dispositivo (lo que, en realidad, ya es sumamente dificultoso en los dispositivos propuestos).

\section{Dispositivo cilíndrico:}

1) El diámetro del dispositivo debe ser adecuado al tamaño del medidor. El prototipo aquí expuesto sirve para un medidor de chorro único.

2) El vidrio de seguridad del prototipo presentado ha sido pegado desde arriba. Para la producción en serie recomendamos introducirlo desde abajo hacia arriba de forma que se encierre completamente, con la finalidad de dificultar aún más el retiro del vidrio de seguridad. Se trata del mismo principio que ya se está aplicando en el dispositivo cuadrado.

\section{Dispositivo cuadrado:}

1) Observamos que la pintura antioxidante usada en nuestro prototipo se desprendía con facilidad, por lo cual se propone poner una capa de barniz encima.

2) El diámetro del dispositivo debe en función del tamaño del medidor. El prototipo aquí expuesto sirve para un medidor de chorro múltiple, por lo que requiere una extensión para ser instalado con un modelo de chorro único; sin embargo, esta problemática se podría evitar fabricando el medidor con un tubo de $31 / 4$ " aprox. en vez del tubo de 4".

\section{Recomendaciones para la instalación}

Dispositivo cilíndrico:

1) Para asegurar que el dispositivo quede bien anclado, es necesario pasar un hierro de construcción de $3 / 8$ " y de $15 \mathrm{~cm}$ de largo por los huecos en la base previstos para tal fin.

2) Para permitir que el medidor entre correctamente en el dispositivo dentro del aro metálico es necesario remover la carcasa y la tapa del reloj del medidor.

\section{Dispositivo cuadrado:}

1) Para que un medidor de chorro único quepa en el dispositivo de las medidas expuestas, se requiere un niple telescópico. Para medidores de chorro múltiple esto no es necesario.

\section{Ambos dispositivos:}

1) Para evitar que el dispositivo sea retirado dentro de las primeras horas después de su instalación, es necesario usar una mezcla de concreto con yeso ("diablo fuerte") o concreto de secado rápido en vez de concreto común de secado lento.

2) Para proteger el medidor en el caso de la inundación de la caja del medidor y evitar el empañamiento de la luneta, recomendamos sellar las aperturas laterales del dispositivo con espuma expansiva de construcción.

\section{Costos aproximados}

Sobre la base de nuestra experiencia con la EPS Grau, estimamos un costo de US\$ 6 a 7 por unidad producida 
en series mayores a 100 unidades; incluyendo la instalación llegamos a un monto de aproximadamente US\$ 10. Teniendo en cuenta el costo del propio medidor protegido, entre US\$ 25 a 30 (chorro único) y US\$ 35 a 45 (chorro múltiple), más el consumo correctamente registrado por el medidor no manipulado, se trata de una alternativa costo-eficiente.

\section{CONCLUSIONES}

Los dispositivos de seguridad disponibles para medidores de agua no protegen el medidor contra todas las formas de manipulación y vandalización.

El uso de dispositivos que protegen solo algunos de los puntos vulnerables de un medidor contra la manipulación lleva a un incremento de la manipulación de medidores en los puntos no protegidos.

Los dos nuevos tipos de dispositivos de seguridad expuestos en el presente artículo protegen el medidor en todos los puntos vulnerables previamente identificados en campo.

Los dos tipos de dispositivos de seguridad presentados pueden ser adecuados al tipo y tamaño del medidor a proteger.

A pesar de su funcionamiento, comprobado en campo, existe potencial de mejora, especialmente en lo que se refiere a la luneta de protección.

Independientemente de los modelos aquí presentados, los resultados del análisis de vulnerabilidad del medidor de agua podrán servir de base para el desarrollo de otros dispositivos de seguridad.

Si bien los dispositivos presentados protegen el medidor en todos sus puntos vulnerables, sigue siendo posible destruir la base en la cual está anclado el medidor y hasta la luneta de vidrio laminado, aunque con mayor fuerza. En este sentido, aparte de dificultar la manipulación del medidor al requerir un mayor esfuerzo y tiempo, sin imposibilitarla, garantiza la visibilidad posterior de la vandalización.

Independientemente de la protección del medidor contra cualquier tipo de manipulación, pueden darse casos de vandalización a los cuales no siempre resistan los dispositivos de seguridad, por lo que se sugiere tomar adicionalmente medidas administrativas y legales para desincentivar esta práctica.

\section{Agradecimientos}

Quisiéramos agradecer al personal que participa en el proyecto de Agua, Saneamiento y Manejo del Recurso Hídrico para Piura, implementado por la Cooperación Económica y la División de Desarrollo la Secretaría de Estado para la Economía (SECO) en el marco de la Cooperación Suiza con la Empresa Prestadora de Servicios de Saneamiento Grau S.A. (EPS Grau); especialmente a Ricardo Rodríguez, coordinador por parte de la consultora AKUT PERÚ, por habernos facilitado los requerimientos administrativos necesarios en tiempo récord, y a Liz Gonzales por los dibujos isométricos para el presente artículo. A Oscar Perfecto (AKUT PERÚ) y a Carlos Boyer (EPS Grau) por sus valiosas ideas en el proceso de desarrollo de los prototipos. A Tania Vega de la EPS Grau por las fotos de campo, a Michaela Ziemendorff por la revisión ortográfica y a Kerry Gubits por los trabajos de traducción. Asimismo a Fernando Carrasco y Gustavo Lindao del banco de medidores de la EPS Grau por su ayuda en la identificación de los tipos y modalidades de manipulación y vandalización de medidores. Finalmente a Carlos Barrientos, Marco Antonio Orrego y Carlos Castillo de la EPS Grau por la fabricación de los prototipos y la primera serie de los dispositivos de seguridad.

\section{REFERENCIAS BIBLIOGRÁFICAS}

Alva Vives, T., J.A. Puelles Barturén y S. Ziemendorff. Serie Gestión Comercial de las EPS. Módulo 01: Actualización dinámica catastral. Programa de Agua Potable y Alcantarillado PROAGUA / GTZ, Lima (Perú), 2006a.

Alva Vives, T., J.A. Puelles Barturén y S. Ziemendorff. Serie Gestión Comercial de las EPS. Módulo 03: Venta de nuevas conexiones. Programa de Agua Potable y Alcantarillado PROAGUA / GTZ, Lima (Perú), 2006b.

Alva Vives, T. Serie Gestión Comercial de las EPS. 
Módulo 05: Herramientas para la optimización del consumo medido. Programa de Agua Potable y Alcantarillado PROAGUA / GTZ, Lima (Perú), 2009.

Cubas Rojas, J.E., J.A. Puelles Barturén y S. Ziemendorff. Serie Gestión Comercial de las EPS. Módulo 02: Regularización masiva de clandestinos. Programa de Agua Potable y Alcantarillado PROAGUA / GTZ, Lima (Perú), 2006.

Danger, G. SmartMeterBlocker.com - Water Meter. L i m a, 2012 . Recuperado de: https://www.youtube.com/watch?v=5R1hJe V0uf8

Medileser. Dispositivo Tipo Copa. Lima (Perú), 2011 a. R e c u p e r a d o d e : http://www.medileser.com.pe/?rule=21:00\&i $\mathrm{dp}=18$

Medileser. Dispositivo Tipo Argolla. Lima (Perú), $2011 \mathrm{~b}$. R e c u p e r a d o d e : http://www.medileser.com.pe/?rule=21:00\&i $\mathrm{dp}=16$

Medileser. Dispositivo Tipo Pulpo. Lima (Perú), $2011 \mathrm{c}$. R e c u p e r a d o d e : http://www.medileser.com.pe/?rule=21:00\&i $\mathrm{dp}=17$

SUNASS (Superintendencia Nacional de Servicios de Saneamiento). "Reglamento de Calidad de la Prestación de Servicios de Saneamiento". El Peruano: 339108-339158. Lima (Perú), 2007.

Ziemendorff, S. "Detección de conexiones clandestinas de agua potable con métodos acústicos un nuevo método y su aplicación en campo", Revista de Investigación para el Desarrollo Sustentable 3(2015): 5-15. 